\title{
Amobarbital sodium and instrumental performance changes following an increase in reward magnitude
}

\author{
JAMES R. ISON and JOHN NORTHMAN, ${ }^{2}$ University of \\ Rochester, Rochester, N.Y.
}

Four groups of hungry rats $(N=12)$ received 54 straight nunway trials at a constant magnitude reward of either 15 or one food pellet, followed by 42 trials in which all groups received 15 pellets reward. Within each reward condition one group received a daily injection of amobarbital sodium before running and one group received a saline injection. The rate of performance increase following the reward shift was not affected by the drug, in contrast to prior observations that the rate of performance decrease following a reward decrement was attenuated by the drug. These data indicate that amobarbital does not degrade S's ability to detect changes in reward stimuli, and support the position that the prior observation resulted because the drug reduces the emotional consequences of reward decrements.

It has been observed that injections of amobarbital sodium eliminated the rapid performance decrement typically found in normal rats following a reduction in reward value (Rosen, Glass, \& Ison, 1967). Prior experiments conducted in a variety of situations have suggested that this drug attenuates the anticipatory emotional consequences of noxious events (Miller, 1964; Ison \& Rosen, 1967) and, thus, it was argued that the effects of reward decrement in the normal animal are determined by such emotionality. But it is necessary to continue to explore other possible effects of amobarbital sodium because, if such effects are demonstrable, they would be confounded with the hypothesized effects on emotionality. Indeed, it may be that nonemotional processes, if they are affected by drug action, are responsible for the rapid change and depression effect in the normal animal.

One candidate for such exploration is the hypothesis that the drug impairs the animal's sensory/perceptual abilities so that manipulations of an external stimulus (such as, but not restricted to, a rewarding stimulus) are less readily detected. Obviously, if the drugged $\mathrm{S}$ does not perceive the change in the reward stimulus then instrumental performance will not be affected by that change. Some support for this hypothesis was obtained in the demonstration that drugged Ss, in contrast to normal Ss, did not approach the changed arm of a T-maze following an initial exploration period (Ison, Glass, \& Bohmer, 1966). Although it may be argued that approach to stimulus change is itself produced by mild emotionality, this observation is certainly consistent with the position that drugged Ss do not detect a change in stimulus conditions.

A critical test of this counter-hypothesis is to determine the effect of the drug on adjustment to an increase in reward value. If drugged Ss do not readily perceive stimulus changes then the rate of performance increment should be attenuated by the drug, parallel to the prior observation that the rate of performance decrement to a reward decrease was so attenuated. If, as initially proposed, the critical drug effect is on emotionality determined by the occurrence of noxious events then no such attenuation should obtain.

\section{SUBJECTS AND APPARATUS}

Male albino rats $(\mathrm{N}=48)$ were obtained from the Holtzman Co., Madison, Wisconsin, at 90 days of age. A straight black runway, 5 in. $\times 5$ in. $x 70$ in., was housed within a sound attenuating hull. A Plexiglas covered channel in the hull directly over the runway permitted uniform dim illumination. Side action retrace doors $11 \mathrm{in}$. from each end of the runway provided start and goal boxes. Photocells on the start door and 6 in., 42 in., and 54 in. from the door, connected to Hunter clocks, measured start, run and goal times. A baffle at the far end of the goal hid a glass coaster which contained the appropriate reward.

$$
\text { PROCEDURE }
$$

Five days prior to running Ss were placed on a 23-h food deprivation schedule ( $60 \mathrm{~min}$ food ad lib per day) and handled for 3 min per day. On the first training day one trial was given, on second day two trials, on third day three trials, and six per day thereafter. The intertrial interval was $3-4 \mathrm{~min}$. A daily injection of amobarbital sodium (IP, $20 \mathrm{mg} / \mathrm{kg}$ ) was given to $24 \mathrm{Ss} 10 \mathrm{~min}$ before running and an equal volume placebo saline injection given to the others. In the first 54 trials (Phase 1) 12 Ss within each injection condition received $15(37 \mathrm{mg})$ food pellets on each trial (Group D-15, Group P-15) and 12 received one pellet (Group D-1, Group P-1). In the subsequent 42 trials (Phase 2) all Ss received 15 pellets. The $\mathrm{S}$ was removed from the goal immediately after consuming the reward.

\section{RESULTS}

Figure 1 depicts start and goal speeds for Phases 1 and 2. In general performance in Phase 1 was greater with the higher reward and the effect of the drug depended on the stage of training and the particular measure. Analyses of variance of start speeds on the first two days of Phase 1 yielded significant drug effects $(F=19.38, \mathrm{~d} f=1 / 44, p<.01)$ and magnitude effects $(F=6.82$, $\mathrm{df}=1 / 44, \mathrm{p}<.05$ ), reflecting the facilitative effects of the higher magnitude of reward and the drug. On goal speeds the effects of drug were not significant $(F=1.16)$, whereas the Magnitude effect $(\mathrm{F}=7.84, \mathrm{df}=1 / 44, \mathrm{p}<.01)$ and a Magnitude by Days effect $(F=9.46, \mathrm{df}=1 / 44, \mathrm{p}<.01)$ were obtained. Analysis of all days of Phase 1 yielded a Magnitude effect $(F=10.40, \mathrm{df}=1 / 44$, $\mathrm{p}<.01)$ and a Magnitude by Days interaction $(\mathrm{F}=3.93$, $\mathrm{df}=8 / 352, \mathrm{p}<.01$ ) on start, and a Magnitude effect on goal $(\mathrm{F}=5.66, \mathrm{df}=1 / 44, \mathrm{p}<.05)$. Start speeds produced an increasing divergence between different magnitudes over days whereas goal speeds showed an initial divergence followed by convergence. Analyses of the last day of Phase 1 yielded a significant effect of magnitude on start behavior $(F=10.68, \mathrm{df}=1 / 44, p<.01)$, but not on goal behavior $(\mathrm{p}>.20)$.

Turning to Phase 2 performance, it is apparent that the performance increases in Groups P-1 and D-1 followed parallel paths; the differences favoring Group D-1 on start speed and Group P-1 on goal speed represent simple continuations of the small Phase 1 differences. Analyses of variance which included the last Phase 1 day and the first two Phase 2 days yielded effects for Days $(p<.01)$ and the Days by Magnitude interaction on both start $(\mathrm{F}=10.52$, df $=2 / 88, \mathrm{p}<.01)$ and goal measure $(\mathrm{F}=5.31$, $\mathrm{d} f=2 / 88, p<.01)$, but no indication that the increase in speed depended on the drug conditions $(p>.20)$. Analyses over all days of Phase 2 yielded only a Days effect on start and goal measures $(F=2.30, \mathrm{df}=6 / 264, \mathrm{p}<.05)$, although a further analysis of

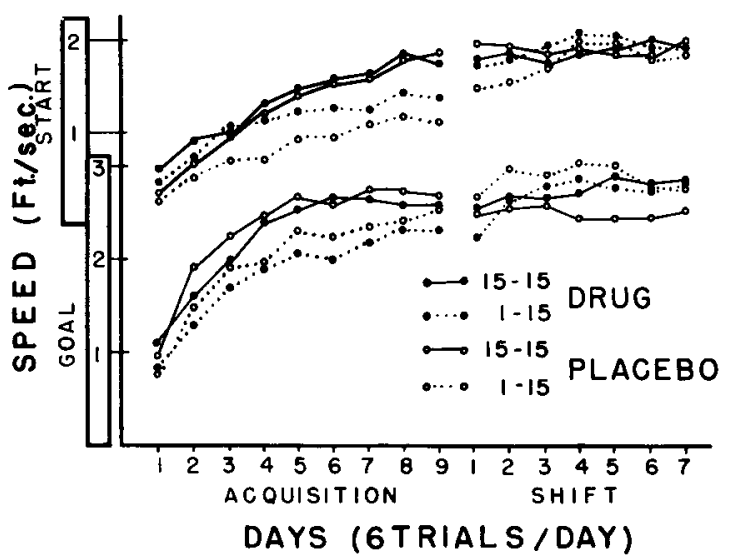

Fig. 1. Start and goal speeds in the 9 pre-shift days (run at either 15 or 1 pellets reward) and the 7 post-shift days (run at 15 pellets reward). 
total speed (the reciprocal of the sum of start, run and goal times) yielded additionally a Magnitude by Days interaction $(\mathrm{F}=2.43$, $\mathrm{df}=6 / 264, \mathrm{p}<.05$ ).

On goal speed Group P-1 exceeded the performance of Group P-15 throughout the shift period. A post-hoc analysis of these two groups yielded a significant Groups effect $(F=4.93, \mathrm{df}=1 / 22$, $\mathrm{p}<.05)$ and a Groups by Days interaction $(\mathrm{F}=14.92$, $\mathrm{df}=6 / 132$, $p<.01)$. Separate analyses of each day yielded a significant Groups effect $(p<.05)$ on Day 4 of Phase 2. This difference should be attributed in part to the decline in Group P-15 over Phase 2.

\section{DISCUSSION}

The failure to observe any effects of amobarbital sodium on the rate of performance increase to a reward increment, in contrast to its striking effect on the rate of performance decline to a reward decrement (Rosen, Glass, \& Ison, 1967), argues against the hypothesis that the drug produces its effect by degrading the organism's detection of changes in reward stimuli. This set of data is generally consistent with the position that the behavioral effects of reward decrements are to be understood in terms of their inhibitory emotional consequences rather than in terms of perceptual contrast mechanisms operating on rewards as stimuli (cf., Ison \& Rosen, 1965).

Three other points may be noted. First, the facilitating effect of amobarbital on start speeds in the early trials is a characteristic of the drug in runway situations that has been attributed to its attenuation of the disturbing effects of fear elicited by novel stimuli (i.e., opening the start door, etc.) in the normal animal (Barry, Wagner, \& Miller, 1962). Second, the apparent decrement in the Phase 1 effectiveness of the different reward magnitudes in the goal measure, in contrast to the start measure, is inconsistent with the simple theoretical position that the effectiveness of rewarding stimuli generalizes back from the goal towards the start. Rather than being an isolated example of this finding it appears frequently in runway performance which has been analyzed into its separate components and where the terminal measure is close to the food cup (e.g., Logan, 1960, p. 54). Third, an elation effect occurred in Group P-1, defined as greater post-shift goal speed in this group compared to Group P-15. The empirical status of elation effects is ambiguous; they are not often demonstrable and when found can sometimes be shown to have an artifactual basis determined by an insufficient number of preshift trials, the lack of proper control groups, different preshift experiences and so on. The present demonstration seems free of these objections but it is clear that the effect was largely determined by a post-shift decrement in the control group in the goal measure, and thus, its theoretical significance should be treated with caution. It may be that concern with elation effects should reflect not excitatory processes occurring in shifted groups but inhibitory processes occurring in non-shifted control groups.

\section{REFERENCES}

BARRY, H., WAGNER, A. R., \& MILLER, N. E. Effects of alcohol and amobarbital on performance inhibited by experimental extinction. Journal of Comparative \& Physiological Psychology, 1962, 55, 464-468.

ISON, J. R., GLASS, D. H., \& BOHMER, H. M. The effects of sodium amytal on the approach to stimulus change. Proceedings of the American Psychological Association, 1966, 2, 5-6.

ISON, J. R., \& ROSEN, A. J. On changes in incentive: some new findings and theoretical speculations. Paper read at Psychonomic Society Meeting, Chicago, Illinois, 1965.

ISON, J. R., \& ROSEN, A. J. The effects of amobarbital sodium on differential instrumental conditioning and subsequent extinction. Psychopharmacologia (Berl.), 1967, 10, 417-425.

LOGAN, F. A. Incentive. New Haven: Yale University Press, 1960.

MILLER, N. E. The analyses of motivational effects illustrated by experiments on amylobarbitone sodium. In H. Steinberg, A. V. S. deReuck, and J. Knight (Eds.), Ciba Foundation Symposium, Jointly with the co-ordinating Committee for Symposia on Drug Action, on Animal Behavior and Drug Action. London: Churchill, 1964. Pp. 1-18.

ROSEN, A. J., GLASS, D. H., \& ISON, J. R. Amobarbital sodium and instrumental performance changes following reward reduction. Psychonomic Science, 1967, 9, 129-130.

\section{NOTES}

1. Supported by research Grants NSF GB 4373 and NSF 7129 to J. R. Ison. The authors are indebted to Mr. Anthony Biglan for running one-third of the Ss.

2. Now at Purdue University, Lafayette, Ind. 